\title{
Internal Partial Discharge and Breakdown Characteristics of Thin Polypropylene Films
}

\author{
R. Shobha and R.S. Nema \\ Indian Institute of Science, Bangalore, India
}

\section{INTRODUCTION}

One of the major causes of failure of polypropylene(PP) film high voltage capacitors is PD (partial discharges). PD can occur in the cavities or gas pockets formed due to variation in tightness of windings, improper impregnation and swelling of the film due to its interaction with the impregnating fluid.The effect of pressure and radial dimensions are important in small cavities mostly because system impregnation is done at low pressures and the cavity size gets reduced .It is probable that with time the cavity pressure will equilibrate with that of the surrounding ambient medium due to diffusion process. An attempt is made here to study the effect of cavity dimensions such as diameter $(0.9 \mathrm{~mm}$ to 3.2 $\mathrm{mm}$ ) and depth $(40 \mu \mathrm{m}$ to $200 \mu \mathrm{m})$ on the various PD characteristics and the related breakdown of thin PP films.

\section{EXPERIMENTAL}

Electrical grade bi-axially oriented PP films of thickness $20 \mu \mathrm{m}$ and 39.8 $\mu \mathrm{m}$ were cut as circular pieces of $110 \mathrm{~mm}$ diameter. Cavity was drilled in the $39.8 \mu \mathrm{m}$ film at its centre using a high-speed PCB-drilling machine. The electrodes used were $2 \pi / 3$ Rogowski profile uniform field brass electrodes of $58 \mathrm{~mm}$ overall diameter and $19 \mathrm{~mm}$ radius of curvature embedded in epoxy (to form $75 \mathrm{~mm}$ overall diameter and $20 \mathrm{~mm}$ overall thickness) except for the flat portion of the electrode surface (exposed effective diameter of the electrode being $48 \mathrm{~mm}$ ). The sample consisted of three layers - the top and bottom layer each of $20 \mu \mathrm{m}$ thickness in contact with the high voltage and ground electrode respectively and the middle layer having a cavity whose diameter/depth is varied.

A matching unit consisting of an RC circuit (R: 1500s, C: $1000 \mathrm{pF}$ ), a coupling capacitor $(600 \mathrm{pF}, 30 \mathrm{kV}$ ), an amplifier (bandwidth $-150 \mathrm{kHz}$ ), power frequency filters at the input and output of the amplifier, an oscilloscope, and other necessary instruments were used for detection and measurement of discharges. The sensitivity of detection was $1 \mathrm{pC}$ with a sample capacitance of $100 \mathrm{pF}$. Electronic counters were used for pulse height analysis.

The inception and extinction voltages wcre measured. The discharge pulse magnitudes and their repetition rates were measured once in four minutes over a period of one hour. The breakdown characteristics of the samples were obtained by the step-stress method. The voltage was raised 
from $1000 \mathrm{~V}$ upto final failure of the sample in steps of $500 \mathrm{~V}$, and held at each step for a finite time interval. The step-time intervals were 10 minutes, 5 minutes, 3 minutes and 1 minute. The total time for failure was recorded using a microsplit-second digital timer.

\section{RESULTS AND DISCUSSIONS}

The inception voltage increases with decreasing cavity diameter for a given depth and increasing cavity depth for a given diameter. The first measurement gives the highest value for inception voltage and subsequent measurements show a reduction in the inception voltage finally stabilizing at a specific value. This stabilized value $V_{i}$, bears a definite relation to the diameter and depth of the cavity (Fig. 1) and is given by,

$$
V_{i}=A\left(d / t^{\prime}\right)^{-B} \text { Volts }
$$

Where $A$ and $B$ are constants, $d$ the cavity diameter, $t$ the cavity depth. The extinction voltages are 12 to 20 per cent lower than the inception voltage.

Since the charge in the gas discharge avalanche must build to a minimum level within the dimensions of the cavity, a relationship exists between the minimum breakdown voltage or the inception voltage and the cavity dimensions (equation 1). When discharges occur in a cavity, the conditions in it may get modified due to the wall charges deposited, which may cause an increase in the probability of finding initiating electrons capable of developing critical avalanches due to field distortions. Also it is probable that products of ionization in the air produced by previous discharges cause a reduction in the breakdown voltage so that the initial inception voltages are the highest. Further, the fact that ultimately inception and extinction voltage stabilize at definite values suggests that the dielectric surface attains a certain optimum charge distribution.

Measurements of discharge magnitudes indicate that a single discharge does not completely discharge the entire cavity. The discharge pulse magnitudes, number and their distribution depend on the diameter as well as depth of the cavity. With time, the rate of change in discharge quantities is less in a smaller diameter cavity. The accumulative repetition rate against apparent charge shows an exponential distribution as compared to an ideal rectangular distribution.

Experimental and calculated values of maximum discharge magnitude for different diameters and depths of the cavity indicate that there is an increasing deviation between the calculated and experimental values as the cavity diameter increases (Table 1 ). This implies that only a part of the cavity and not the whole of it discharges. It must be said that this conclusion is based on the assumption that the cavity becomes 
conducting after the discharge i.e. the voltage drop across the cavity is equal to the breakdown voltage of the cavity. Hence the effective diameter for the discharge can be calculated as follows,

Effective diameter of the discharge,

$$
\mathrm{d}_{\text {effective }}=\mathrm{d}_{\text {cavity }}\left[\mathrm{Q}_{\text {experimenta! }} / \mathrm{Q}_{\text {calculated }}\right]^{1 / 2}
$$

where $Q$ is the charge magnitude

The effective diameters of discharge calculated are between $0.50 \mathrm{~mm}$ and $0.83 \mathrm{~mm}$. The increase in the effective diameter with increasing diameter of the cavity is probably due to increased capacitance and hence the stored energy. In samples with larger cavity diameter the area available for discharges is large. The discharges occur preferentially between sites which are devoid of the effect of surface charges left behind by previous discharges.The discharges can follow paths of successively increasing lengths. Hence the number of smaller as well as larger magnitude pulses are higher. It is expected that for larger cavity diameter, a single discharge can cause wall charges to collect only on certain areas of the cavity wall facing the channel and not all over the wall. This should explain for the greater variation in the number of pulses with increasing cavity dimension.It is probable that the greatest discharges occuring between the central parts of the cavity or tangential are less affected by the wall charges and hence vary less with time. The variations in discharge quantities with time could also be due to changes in the gas pressure as well as composition of the discharge products.

The inverse power-law model has been frequently used to estimate lifetimes of insulating materials under voltage stress. In the simplest form this model is represented by the equation,

$$
\mathrm{t}(\mathrm{E})^{\mathrm{n}}=\mathrm{k}
$$

where $t$ is the time to failure at voltage stress $E$, ' $n$ ' and ' $k$ ' are constants. Starr and Endicott [1] proposed the concept of equivalent damage to estimate the life at constant stress from that on linearly increasing progressive stress. They found that the data fit a straight line on a log-log paper for progressive stress and hence suggested the applicability of inverse power-law model for progressive stress also. Assuming a similar cumulative nature of damage to the insulation for the step-stress, the cumulative damage will be sum of the damages at each voltage level, given by,

$$
t(E)^{n}=\Sigma t_{s}\left(E_{s}\right)^{n}+t_{s+1}\left(E_{s+1}\right)^{n}
$$

$t_{s} E_{s}$ is a product of time and voltage stress at each fully completed step, $t_{s+1}$ is the time to failure on the uncompleted $E_{s+1}$ step. Comparison of equations (3) and (4) indicate that the right hand side of equation (4) is a constant and is independent of the time interval ts chosen, provided 
the ageing mechanism has not changed over the covered range. The advantage of using the step-stress method is that the times to failure is considerably reduced [2].

The breakdown stress against time to failure, E-t characteristics, is shown in Fig.2. The ' $n$ ' values obtained from these curves are tabulated in Table-2. The ' $n$ ' values as well as the discharge quantities vary with cavity dimensions. An attempt has been made to fit an equation of the form $n$ $=A^{\prime}$ exp- $B^{\prime}\left(Q_{m}\right)$, where $A^{\prime}$ and $B^{\prime}$ are constants and $Q_{m}$ is the maximum discharge magnitude. On extrapolation, this equation gives ' $n$ ' value of 11.3 for a permissible discharge magnitude of $10 \mathrm{pC}$. Krishnan and Nema [3] in their work on surface PD ageing of PP films have obtained ' $n$ ' value corresponding to $10 \mathrm{pC}$ as 11.8 . The total discharge quantity/second $Q_{t}$ upto failure correlates with the time to failure $(L)$ in the form $L=a\left(Q_{t}\right)^{-b}$ and is shown in Fig.3. This indicates that $Q_{t}$ seems to be an important factor in the degradation of PP films. This aspect falls in line with the observations of Krishnan and Nema [3] where a similar relation for $\mathrm{L}$ and $\mathrm{Q}_{t}$ is obtained for surface discharge studies, and of Dakin [4] where it is stated that the rate of material degradation is proportional to the total number of discharges per second. It has also been found that for a given diameter and depth of the cavity, the ' $k$ ' values corresponding to 10 minutes, 5 minutes, 3 minutes and 1 minute step-time intervals are all of the same order. Hence this suggests that the step-stress method can be used for a quick determination of the constants of the inverse power-law model for life characteristics in the presence of PD.

\section{REFERENCES}

[1] W.T. Starr and H.S. Endicott :"Progressive Stress- A New Accelerated Approach to Endurance", Trans. AIEE, PAS 80, 1961, 575.

[2] J.R. Laghari, P. Cygan and W. Khechen: "A Short Method of Estimating Lifetime of Polypropylene Film using Step-Stress Tests", IEEE Trans. on Elec. Insul. 25(6), 1990, 1180.

[3] V. Krishnan and R.S. Nema : "A Study of Short-term Partial Discharge Ageing of Polypropylene Film", IEEE Trans. Elec. Insul. 24(6), 1989, 1133.

[4] T.W. Dakin : "Theory of Voltage Endurance", Addendum to Conf. Proc. CEIDP, 1984.

\section{ACKNOWLEDGEMENTS}

Thanks are due to the authorities of Indian Institute of Science and Central Power Research Institute, Bangalore, India, for granting permission to publish this work. 


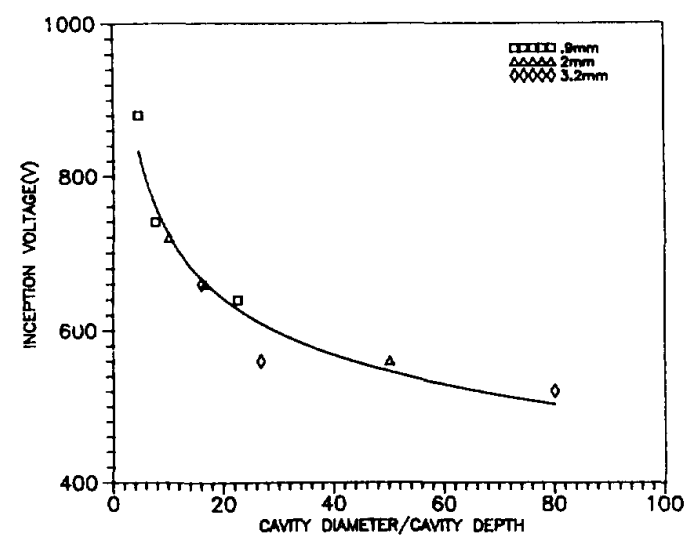

Fig. 1 : Variation of inception voltage with cavity diameter/depth.

Table 1 : Calculated values of Maximum Discharge Magnitude

\begin{tabular}{cccc}
\hline $\begin{array}{c}\text { Cavity } \\
\text { depth } \\
(\mu \mathrm{m})\end{array}$ & $\begin{array}{c}\text { Cavity } \\
\text { diameter } \\
(\mathrm{mm})\end{array}$ & $\begin{array}{c}\mathbf{Q}_{\mathrm{cal}} \\
(\mathrm{pC})\end{array}$ & $\begin{array}{c}\mathbf{Q}_{\text {expl }} \\
\mathbf{Q}_{\mathrm{cal}}\end{array}$ \\
\hline & 0.9 & 198 & 0.31
\end{tabular}

$\begin{array}{llll}40 & 2.0 & 976 & 0.13\end{array}$

$----\frac{3.2}{0.9}--\frac{2499}{330}--\frac{0.07}{0.40}$

$\begin{array}{llll}120 & 2.0 & 1631 & 0.11\end{array}$

$----\frac{3.2}{0.9}--\frac{4175}{434}--\frac{0.05}{0.40}$

$\begin{array}{llll}200 & 2.0 & 2142 & 0.10\end{array}$

\begin{tabular}{lll}
3.2 & 5483 & 0.04 \\
\hline
\end{tabular}

$Q_{\mathrm{cal}}=Q_{\mathrm{cal}} \mathrm{culated}$

$Q_{\exp }=Q_{\text {expertrnental }}$
Table 2 : Constant ' $n$ ' of the inverse power-law model

\begin{tabular}{|c|c|c|c|}
\hline \multirow{2}{*}{$\begin{array}{l}\text { Cavity } \\
\text { depth } \\
(\mu \mathrm{m})\end{array}$} & \multicolumn{3}{|c|}{ Cavity diameter - mm } \\
\hline & 0.9 & 2.0 & 3.2 \\
\hline 80 & 9.02 & 9.23 & 8.88 \\
\hline 120 & 7.03 & 5.99 & 6.60 \\
\hline 200 & 5.59 & 5.53 & 6.50 \\
\hline
\end{tabular}




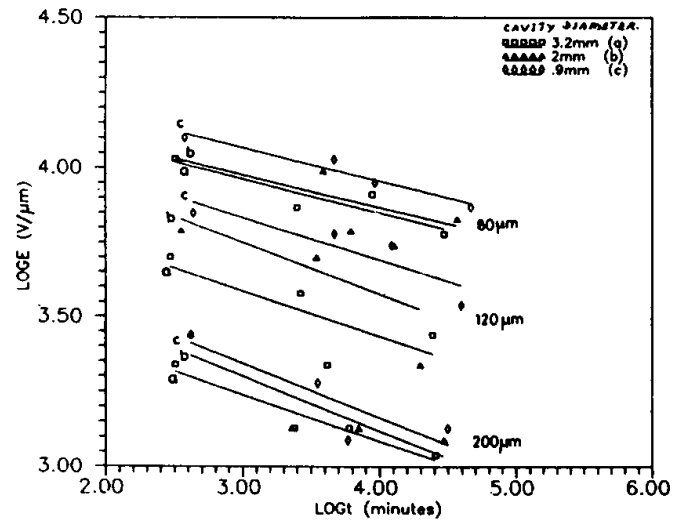

Fig. 2 : Effect of cavity dimensions on the E-t characteristics.

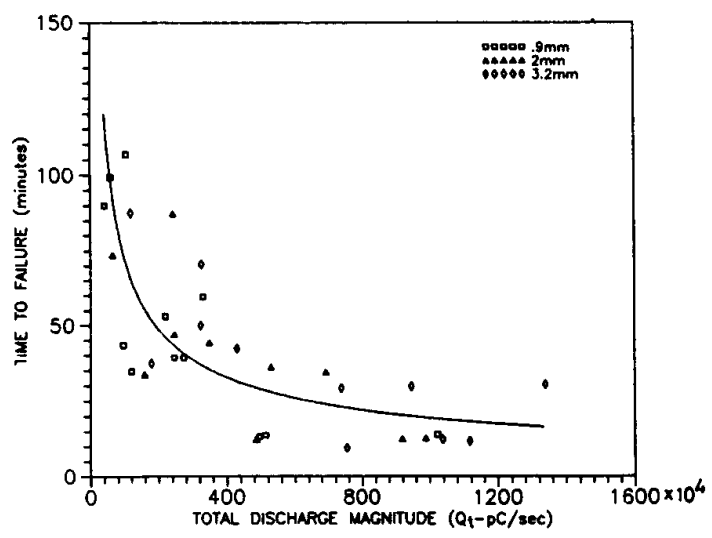

Fig. 3 : Variation of time to failure with total discharge magnitude. 